\title{
Cr-Salen mediated asymmetric epoxidation of alkenes: rational complex design and substrate scope of catalyst
}

\author{
Eoin F. Clarke, Eoghan M. McGarrigle, and Declan G. Gilheany* \\ Chemistry Department, Centre for Synthesis and Chemical Biology, Conway Institute of \\ Biomolecular and Biomedical Sciences, University College Dublin, Belfield, Dublin 4, Ireland \\ E-mail: declan.gilheany@ucd.ie
}

This paper is dedicated to the memory of Kevin Phelan

(received 07 Sept 04; accepted 14 Oct 04; published on the web 26 Oct 04)

\begin{abstract}
Recent explorations of the structure space of $\mathrm{Cr}$ (salen) complexes have led to the rational design of improved catalysts for the asymmetric epoxidation of alkenes. The synthesis of these catalysts is presented and their substrate scope detailed. Catalyst 3a, in combination with triphenylphosphine oxide, gives the highest enantiomeric excesses achieved to date in both stoichiometric and catalytic epoxidation with $\mathrm{Cr}$ (salen) complexes.
\end{abstract}

Keywords: Asymmetric, epoxidation, salen, chromium, electronic tuning, asymmetric catalysis

\section{Introduction}

Metal(salen)-mediated epoxidation continues to attract a great deal of attention ${ }^{1-3}$ and research continues apace into both mechanistic details ${ }^{4-10}$ and practical improvements. ${ }^{11-19}$ Chromium(salen) complexes have been shown to be capable of mediating a variety of other reactions $^{20-33}$ as well as the asymmetric epoxidation (AE) of alkenes.34-37 We discovered that the Cr-system shows high enantioselectivity for the conversion of trans-1,2-disubstituted alkenes (trans-alkenes henceforth) in contrast to almost all known Mn(salen) systems. ${ }^{38,} 39$ Also in contrast to the $\mathrm{Mn}$ (salen) system, with $\mathrm{Cr}$ (salen) it is possible to isolate the metal-oxo complex proposed as the active oxidant in both systems. This has allowed us to study the reaction of the alkene with the (salen) $\mathrm{Cr}^{\mathrm{V}}=\mathrm{O}$ species in isolation from other aspects of the catalytic cycle.
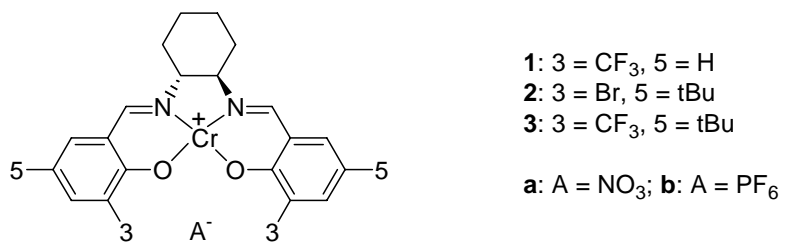
We have reported detailed studies of the effect on AE of various substituents at all positions on the arene ring of the salen ligand ${ }^{40}$ which culminated in complex 1a. ${ }^{41,42}$ Recently, an exploration of salen substituent effects led to the discovery of complex $\mathbf{2} \mathbf{b}$, which was also an effective mediator of epoxidation. ${ }^{43}$ We envisaged that, by incorporating the beneficial substituents from $\mathbf{1 a}$ and $\mathbf{2 b}$ into a new catalyst $\mathbf{3}$, we could obtain further improvements in the asymmetric epoxidation of unfunctionalised alkenes. The new catalyst was expected to be a less reactive but more selective epoxidising agent. We now describe the synthesis of 3a,b and their use in the $\mathrm{AE}$ of a variety of classes of alkenes

\section{Results and Discussion}

Scheme 1 shows the synthetic route adopted for the synthesis of 3a,b. After unsuccessful attempts to introduce the trifluoromethyl group into the benzene ring late in the synthesis, we adopted the methods previously used for the synthesis of 3-trifluoromethylsalicylaldehyde. ${ }^{41}$ The synthesis of phenol 5 had been described previously by Stokker et al ${ }^{44}$ The protection, formylation, and deprotection steps proceeded in good yield. ${ }^{45}$ Contrary to our previous experience, ${ }^{41}$ the use of $N, N, N^{\prime}, N^{\prime}$-tetramethylethylenediamine (TMEDA) was found to be essential to achieve a selective formylation - a complicated mixture resulted in its absence. Care had to be taken also to prevent decomposition of the delicate trifluoromethyl phenol derivative 7 which is sensitive to acid and base. ${ }^{46,47}$ After some experimentation, we found that $3 \%$ TFA in $\mathrm{CH} 2 \mathrm{Cl} 2$ removed the protecting group without causing the product to decompose.
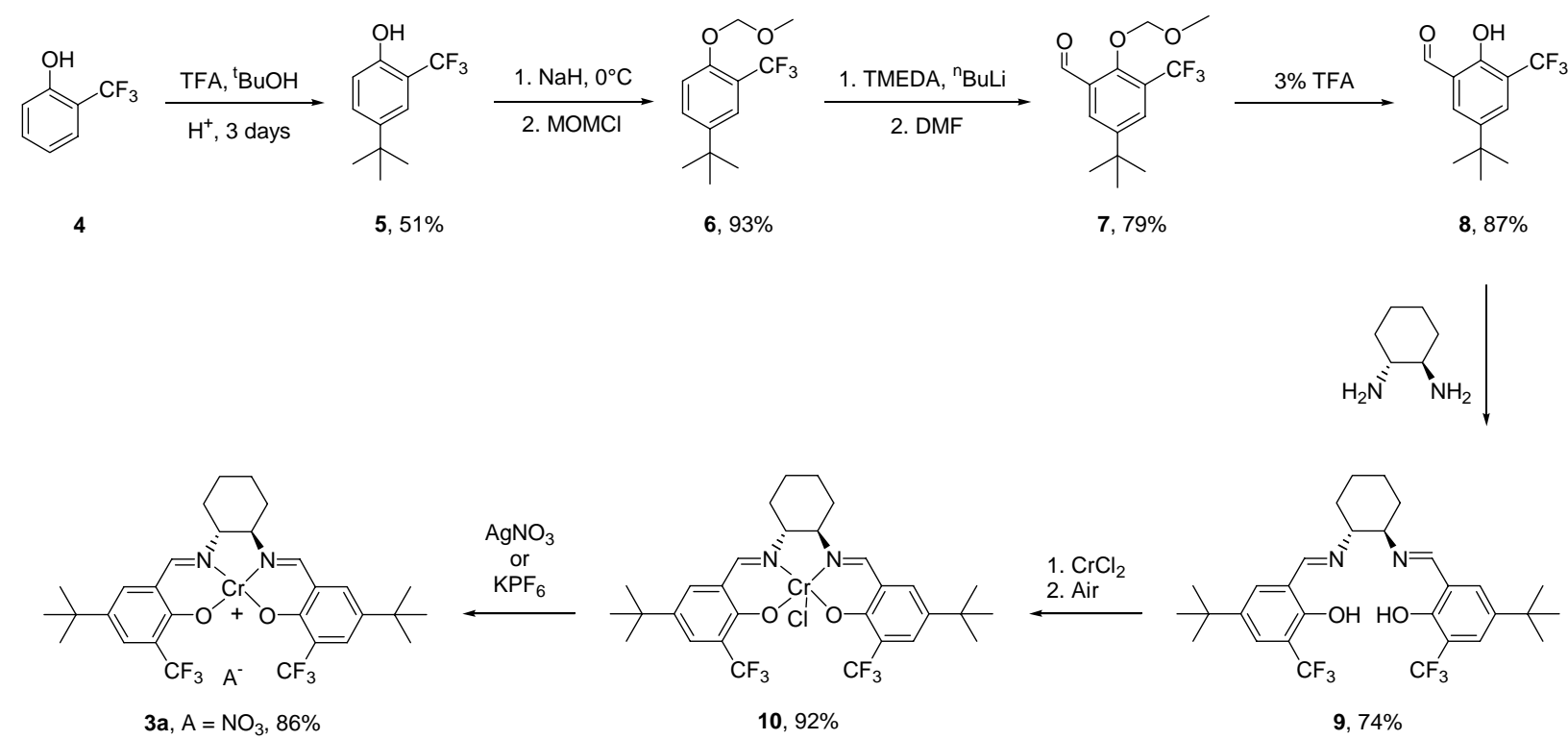

Scheme 1. Synthesis of $\mathrm{Cr}($ salen) complexes (overall yield $=18-19 \%$ ). 
With the requisite salicylaldehyde $\mathbf{8}$ in hand, the synthesis of 3a,b was achieved in good yield using standard methods. ${ }^{41}$ The paramagnetic complexes were characterised by IR and ESMS. Elemental analyses were not satisfactory, however this is not unusual for these complexes ${ }^{26}$ and epoxidation results were reproducible from batch to batch.

We first tested our new catalysts in the stoichiometric epoxidation of trans- $\beta$-methylstyrene (our standard test substrate) by $\mathrm{O}=\mathrm{Cr}$ (salen) (see Table 1). Gratifyingly, the catalysts lived up to our expectations, exhibiting higher enantioselectivity than any previous $\mathrm{Cr}$ (salen) catalyst in the epoxidation of this substrate. The epoxide is stable in the presence of $\mathrm{Cr}^{\mathrm{III}}(\mathrm{salen})^{+}$and $\mathrm{O}=\mathrm{Cr}^{\mathrm{V}}$ (salen) ${ }^{+}$, thus enantioselectivity is not a result of kinetic resolution. The effect of changing counterion was unusually large, nitrate was found to be significantly better than hexafluorophosphate. The addition of $\mathrm{Ph}_{3} \mathrm{PO}$ was found to improve the ees obtained, in line with previous results. The yields in stoichiometric mode were low, however this is not unexpected. ${ }^{43}$

Table 1. Epoxidation of trans- $\beta$-methylstyrene in acetonitrile with $\mathrm{PhIO}$ as oxidant at $0^{\circ} \mathrm{C}^{\mathrm{a}}$

\begin{tabular}{lllllll}
\hline No. & Catalyst & Additive & \multicolumn{2}{l}{ Stoichiometric $^{\mathrm{b}}$} & \multicolumn{2}{l}{ Catalytic $^{\mathrm{c}}$} \\
\hline & & & Ee (yield) & Time (h) & Ee (yield) & Time (h) \\
1 & 3a & None & $92(15)$ & 24 & $76(8)$ & 42 \\
2 & 3a & $\mathrm{Ph}_{3} \mathrm{PO}$ & $94(23)$ & 24 & $88(16)$ & 42 \\
3 & 3a & $\mathrm{Ph}_{3} \mathrm{PO}$ & - & 24 & $-15(3) \mathrm{d}$ & 18 \\
4 & 3b & None & $79(18)$ & 3 & - & \\
5 & 3b & $\mathrm{Ph}_{3} \mathrm{PO}$ & $89(27)$ & 3 & - & \\
\hline
\end{tabular}

${ }^{\mathrm{a}}$ See experimental section for full details. ${ }^{\mathrm{b}} 1.0$ equiv. of $\mathrm{O}=\mathrm{Cr}$ (salen) w.r.t. substrate. ${ }^{\mathrm{c}} 0.10$ equiv. of $\mathrm{Cr}$ (salen) and 1.5 equiv. of $\mathrm{PhIO}$ w.r.t. substrate. ${ }^{\mathrm{d}}$ with 5.0 equiv. of $\mathrm{NaOCl}(0.55 \mathrm{M}$ in $0.05 \mathrm{M}$ $\mathrm{NaHPO}_{4}$ ) as oxidant.

The most notable result in Table 1 is that obtained in catalytic mode $\left(10 \mathrm{~mol} \% \mathrm{Cr}^{\mathrm{III}}\right.$ (salen)) with $\mathrm{Ph}_{3} \mathrm{PO}$ additive, which is the highest ee $(88 \%)$ obtained thus far under catalytic conditions with $\mathrm{Cr}$ (salen) complexes. However it is still slightly lower than in stoichiometric mode, as had been observed previously with 1a. ${ }^{41}$ Also the yields were unexpectedly low, usually the yield in catalytic mode is significantly higher than in stoichiometric mode (71\% vs $45 \%$ with 1a). The very low catalytic yield is probably due to disproportionation of $\mathrm{PhIO}$ over the extended reaction times necessary for this slower catalyst. ${ }^{48}$ In line with previous results, ${ }^{41}$ the use of $\mathrm{NaOCl}$ as terminal oxidant resulted in a poor ee (entry 3 ), however, the sense of the enantioselectivity was reversed suggesting a different oxidation pathway. ${ }^{43}$ We have also rationalised the reduction in ee relative to stoichiometric mode in terms of a second oxidation cycle being in operation under catalytic conditions. ${ }^{41,43}$ Evidence for the presence of multiple oxidation cycles has been reported for $\mathrm{Mn}$ (salen)-, $\mathrm{Mn}$ (corrole)- and $\mathrm{Fe}$ (porphyrin)-catalysed epoxidations. ${ }^{49-53}$ Bryliakov and Talsi $^{10}$ have recently reported evidence supporting the presence of two discrete (salen)chromium-oxo species starting from $\mathrm{Cr}$ (salen) chloride complexes and iodosylbenzene: a 
monomeric [(salen) $\mathrm{Cr}^{\mathrm{V}}=\mathrm{O}^{+}$and a dimeric mixed valent $\mathrm{Cr}^{\mathrm{III}} \mathrm{Cr}^{\mathrm{V}}$-oxo species. They proposed that only the former species was acting as an oxidant under their conditions, while the latter species acted as a reservoir. ${ }^{10}$ The nature of the proposed second oxidant and/or oxidation cycle in our system is currently under investigation in our laboratory and will be the focus of a future report.

Having established that 3a was the better catalyst we then examined its substrate scope. Examples of terminal mono-substituted, terminal di-substituted, cis- and trans-1,2-disubstituted alkenes, were all examined. Table 2 shows results for those substrates where epoxide was formed as product. Unfortunately, the excellent selectivity of the catalyst in the case of trans- $\beta$ methylstyrene did not extend to other substrates. Only trans-stilbene and cis- $\beta$-methylstyrene gave ees greater than $50 \%$, which is a significant improvement for the latter substrate in $\mathrm{Cr}$ (salen)-mediated AE. Yields were poor in all cases. The attempted epoxidation of $\alpha$ methylstyrene resulted in a complicated mixture with no epoxide or starting material present. trans-Methyl cinnamate was recovered unchanged after 48 hours under stoichiometric conditions.

Table 2. Epoxidation of alkenes using 3a and $\mathrm{PhIO}$ in acetonitrile at $0^{\circ} \mathrm{C}^{\mathrm{a}}$

\begin{tabular}{|c|c|c|c|c|c|c|}
\hline \multirow[t]{2}{*}{ No. } & \multirow[t]{2}{*}{ Substrate } & \multirow[t]{2}{*}{ Additive } & \multicolumn{2}{|c|}{ Stoichiometric } & \multicolumn{2}{|l|}{ Catalytic } \\
\hline & & & Ee (yield) & Time (h) & Ee (yield) & Time (h) \\
\hline 1 & trans- $\beta$-Methylstyrene & None & $92(15)$ & 24 & $76(8)$ & 42 \\
\hline 2 & trans- $\beta$-Methylstyrene & $\mathrm{Ph}_{3} \mathrm{PO}$ & $94(23)$ & 24 & $88(16)$ & 42 \\
\hline 3 & cis- $\beta$-Methylstyrene & None & $57(1)$ & 24 & $44(17)$ & 42 \\
\hline 4 & cis- $\beta$-Methylstyrene & $\mathrm{Ph}_{3} \mathrm{PO}$ & $51(3)$ & 24 & $41(21)$ & 42 \\
\hline 5 & Styrene & None & $\mathrm{NR}^{\mathrm{b}}$ & 42 & - & - \\
\hline 6 & Styrene & $\mathrm{Ph}_{3} \mathrm{PO}$ & $7\left(\mathrm{ND}^{\mathrm{c}}\right)$ & 42 & - & - \\
\hline 7 & trans-Stilbene & None & $50(<10)$ & 42 & - & - \\
\hline 8 & trans-Stilbene & $\mathrm{Ph}_{3} \mathrm{PO}$ & $58(10)$ & 42 & - & - \\
\hline
\end{tabular}

${ }^{\mathrm{a}}$ See Table 1 for general details. Entries $1 \& 2$ are taken from Table $1 .{ }^{\mathrm{b}} \mathrm{NR}=$ no reaction. ${ }^{\mathrm{c}} \mathrm{ND}=$ not determined.

\section{Conclusions}

A rationally designed, novel $\mathrm{Cr}$ (salen) complex has been synthesised and tested in the epoxidation of a range of alkenes. The highest ever selectivity for the AE of trans- $\beta$ methylstyrene by a $\mathrm{Cr}$ (salen) complex was achieved but only moderate to poor selectivity with other substrates. The differences in results between catalytic and stoichiometric modes are suggested to be due to the presence of a second oxidation cycle. 


\section{Experimental Section}

General Procedures. All chemicals were available from Aldrich and used as received unless stated. 2-Trifluoromethylphenol was obtained from Fluorochem Ltd. and used as received. transCyclohexane-1,2-diamine was purchased in racemic form and resolved using literature methods. ${ }^{54}$ Iodosylbenzene was synthesised using literature procedures. ${ }^{55}$

4-tert-Butyl-2-trifluoromethylphenol (5). A modified version of the procedure of Stokker et al. was used. ${ }^{44}$ To a solution of 2-trifluoromethylphenol $(24.0 \mathrm{~g}, 148 \mathrm{mmol})$ and tert-butanol $(11.5 \mathrm{~g}, 155 \mathrm{mmol})$ in trifluoroacetic acid (TFA) $(100 \mathrm{ml})$ was added concentrated sulphuric acid $(2 \mathrm{ml})$. The colourless solution was stirred for 3 days at room temperature, during which time a dark yellow colour developed. The reaction mixture was concentrated under vacuum to yield a dark green oil $(26.4 \mathrm{~g})$ which was dissolved in $\mathrm{CH}_{2} \mathrm{Cl}_{2}(150 \mathrm{ml})$. This solution was then washed with water $(150 \mathrm{ml})$, saturated sodium bicarbonate $(3 \times 150 \mathrm{ml})$ and brine $(150 \mathrm{ml})$. After drying over $\mathrm{Na}_{2} \mathrm{SO}_{4}$, the solvent was removed under vacuum to yield a light green coloured oil which solidified on standing. The resulting solid was recrystallised from pet. spirits $\left(40-60^{\circ} \mathrm{C}\right)$ to yield white crystals $(16.48 \mathrm{~g}, 51 \%)$ : Mp 57-61 ${ }^{\circ} \mathrm{C} ;{ }^{1} \mathrm{H} \mathrm{NMR}\left(300 \mathrm{MHz}, \mathrm{CDCl}_{3}\right) \delta 7.49(\mathrm{~d}, 1 \mathrm{H}, J=2.3$ $\mathrm{Hz}, \mathrm{ArH}), 7.43$ (dd, 1H, $J=8.6 \mathrm{~Hz}, 2.3 \mathrm{~Hz}, \mathrm{ArH}), 6.88$ (d, $1 \mathrm{H}, J=8.6 \mathrm{~Hz}, \mathrm{ArH}), 5.62(\mathrm{~s}, 1 \mathrm{H}$, $\mathrm{OH}), 1.30\left(\mathrm{~s}, 9 \mathrm{H},{ }^{\mathrm{t}} \mathrm{Bu}\right)$; MS (GCMS) $\mathrm{m} / z$ (\% relative intensity) $218.2\left(\mathrm{M}^{+}, 40\right), 203.2\left(\mathrm{M}-\mathrm{CH}_{3}\right.$, 100), 183.2 (90), 155.1 (30), 127.1 (20).

4-tert-Butyl-1-methoxymethoxy-2-trifluoromethylbenzene (6). Sodium hydride (1.38 g of a $60 \%$ dispersion in mineral oil, $34.5 \mathrm{mmol}$ ) was washed with hexane and transferred to a 3-neck $100 \mathrm{ml} \mathrm{RB}$ flask under an atmosphere of $\mathrm{N}_{2}$. After addition of anhydrous DMF $(11 \mathrm{ml})$, the slurry was cooled with stirring to $0^{\circ} \mathrm{C}$. To the resulting grey suspension, a solution of $5(5.00 \mathrm{~g}$, $22.9 \mathrm{mmol})$ in anhydrous DMF $(6 \mathrm{ml})$ was added dropwise at such a rate that the evolution of hydrogen did not become too vigorous. After complete addition the ice bath was removed and the reaction mixture was stirred for $1 \mathrm{~h}$. Chloromethylmethyl ether $(2.6 \mathrm{ml}, 34 \mathrm{mmol})$ was then added dropwise and the resulting solution was stirred overnight. Ice/water $(50 \mathrm{ml})$ was added cautiously and the mixture extracted with $\mathrm{Et}_{2} \mathrm{O}(3 \times 50 \mathrm{ml})$. The combined $\mathrm{Et}_{2} \mathrm{O}$ extracts were washed with $\mathrm{NaOH}$ solution $(2 \mathrm{M}, 50 \mathrm{ml})$, hydrochloric acid $(2 \mathrm{M}, 50 \mathrm{ml})$, and brine $(50 \mathrm{ml})$. The solution was dried over $\mathrm{MgSO}_{4}$ and the solvent was removed in vacuo to yield a colourless liquid $(5.57 \mathrm{~g}, 93 \%)$ which was used in the next step without further purification: ${ }^{1} \mathrm{H}$ NMR $(300 \mathrm{MHz}$, $\left.\mathrm{CDCl}_{3}\right) \delta 7.57(\mathrm{~d}, 1 \mathrm{H}, J=2.3 \mathrm{~Hz}, \mathrm{ArH}), 7.47(\mathrm{dd}, 1 \mathrm{H}, J=2.3 \mathrm{~Hz}, J=8.6 \mathrm{~Hz}, \mathrm{ArH}), 7.15(\mathrm{~d}, 1 \mathrm{H}$, $J=8.6 \mathrm{~Hz}, \mathrm{ArH}), 5.24\left(\mathrm{~s}, 2 \mathrm{H}, \mathrm{CH}_{2}\right), 3.50\left(\mathrm{~s}, 3 \mathrm{H}, \mathrm{CH}_{3}\right), 1.31\left(\mathrm{~s}, 9 \mathrm{H},{ }^{t} \mathrm{Bu}\right) ;{ }^{13} \mathrm{C} \mathrm{NMR}(75 \mathrm{MHz}$, $\left.\mathrm{CDCl}_{3}\right) \delta 152.9,143.2,130.2,123.9\left(\mathrm{q},-\mathrm{CF}_{3}, J_{\mathrm{C}-\mathrm{F}}=272 \mathrm{~Hz}\right), 124.0,119.0\left(\mathrm{q}, J_{\mathrm{C}-\mathrm{F}}=30.5\right) 115.2$, $94.5\left(-\mathrm{CH}_{2}-\right), 56.4\left(-\mathrm{OCH}_{3}\right), 34.5\left(-\mathrm{CMe}_{3}\right), 31.5\left(-\mathrm{CH}_{3}\right)$; MS (GCMS) $\mathrm{m} / \mathrm{z}$ (\% relative intensity) $262.2\left(\mathrm{M}^{+}, 50\right), 247.2$ (25), 232.2 (10), $217.2\left(\mathrm{M}-\mathrm{C}_{2} \mathrm{H}_{5} \mathrm{O}, 100\right), 201.2$ (10) 183.1 (20); IR $\left(\mathrm{KBr}, \mathrm{cm}^{-1}\right) 2961,1619,1589,1508,1423,914,741$;

5-tert-Butyl-2-methoxymethoxy-3-trifluoromethylbenzaldehyde (7). To a solution of 6 $(8.41 \mathrm{~g}, 32.1 \mathrm{mmol})$ in dry hexane $(75 \mathrm{ml})$ under an atmosphere of $\mathrm{N}_{2}$ was added TMEDA 
(4.9 ml, $32 \mathrm{mmol})$ via syringe. The solution was then cooled to $0^{\circ} \mathrm{C}$ and $n \mathrm{BuLi}(20 \mathrm{ml}$ of a $1.6 \mathrm{M}$ solution in hexanes, $32 \mathrm{mmol}$ ) was added dropwise via syringe. The resulting thick grey solution was stirred for $2 \mathrm{~h}$ at $0^{\circ} \mathrm{C}$ followed by addition of dry DMF $(2.72 \mathrm{ml}, 35.1 \mathrm{mmol})$. The solution was then stirred for $0.5 \mathrm{~h}$ at $0^{\circ} \mathrm{C}$ followed by $1 \mathrm{~h}$ at room temperature. Following the addition of $1 \mathrm{M} \mathrm{HCl}(50 \mathrm{ml})$ the mixture was extracted with $\mathrm{Et}_{2} \mathrm{O}(3 \times 50 \mathrm{ml})$. The combined ether extracts were then washed with brine $(50 \mathrm{ml})$, dried over $\mathrm{MgSO}_{4}$, and the solvent was removed under reduced pressure to yield a clear liquid, which was distilled under vacuum (7.39 g, 79\%): Bp 95$96^{\circ} \mathrm{C}(0.1 \mathrm{~mm} \mathrm{Hg}) ;{ }^{1} \mathrm{H}$ NMR $\left(300 \mathrm{MHz}, \mathrm{CDCl}_{3}\right) \delta 10.31(\mathrm{~s}, 1 \mathrm{H}, \mathrm{CHO}), 8.06(\mathrm{~d}, 1 \mathrm{H}, J=8.6 \mathrm{~Hz}$, ArH), $7.86(\mathrm{~d}, 1 \mathrm{H}, J=8.6 \mathrm{~Hz}, \mathrm{ArH}), 5.10\left(\mathrm{~s}, 2 \mathrm{H}, \mathrm{CH}_{2}\right), 3.63\left(\mathrm{~s}, 3 \mathrm{H}, \mathrm{CH}_{3}\right), 1.36\left(\mathrm{~s}, 9 \mathrm{H},{ }^{\mathrm{t}} \mathrm{Bu}\right)$; IR $\left(\mathrm{KBr}, \mathrm{cm}^{-1}\right)$ 2965, 1697, 1608, 1481, 1326, 924; Anal. calcd for $\mathrm{C}_{14} \mathrm{H}_{17} \mathrm{~F}_{3} \mathrm{O}_{3}: \mathrm{C}, 57.93 ; \mathrm{H}, 5.90$; F, 19.63. Found: C, 58.19; H, 6.06; F, 20.07.

5-tert-Butyl-2-hydroxy-3-trifluoromethylbenzaldehyde (8). Aldehyde 7 (7.07 g, 24.4 mmol) was dissolved in a $3 \%$ solution of TFA in $\mathrm{CH}_{2} \mathrm{Cl}_{2}(180 \mathrm{ml})$. The solution was stirred and monitored by TLC for the disappearance of starting material. When no starting material remained, the solvent was removed in vacuo to yield a clear solution which, on standing, solidified to yield a low melting solid $(5.19 \mathrm{~g}, 87 \%) .{ }^{1} \mathrm{H} \mathrm{NMR}\left(300 \mathrm{MHz}, \mathrm{CDCl}_{3}\right) \delta 11.56(\mathrm{~s}, 1 \mathrm{H}$, $\mathrm{OH}), 9.97$ (s, 1H, CHO), 7.84 (d, 1H, $J=1.9 \mathrm{~Hz}, \mathrm{ArH}), 7.76$ (d, 1H, $J=1.9 \mathrm{~Hz}, \mathrm{ArH}), 1.38$ (s, $\left.9 \mathrm{H},{ }^{\mathrm{t}} \mathrm{Bu}\right) ;{ }^{13} \mathrm{C} \mathrm{NMR}\left(75 \mathrm{MHz}, \mathrm{CDCl}_{3}\right.$ ) $\delta 196.8$ (ArCHO), 157.7, 142.8, $134.0(\mathrm{ArCH}), 131.5$ (q, $\left.J_{\mathrm{C}-\mathrm{F}}=5.0 \mathrm{~Hz}, \mathrm{ArCH}\right), 123.4\left(\mathrm{q},-\mathrm{CF}_{3}, J_{\mathrm{C}-\mathrm{F}}=273 \mathrm{~Hz}\right), 121.1,118.8\left(\mathrm{q}, J_{\mathrm{C}-\mathrm{F}}=31 \mathrm{~Hz}, \mathrm{ArC}_{3}\right), 34.5$ $\left(-\mathrm{CMe}_{3}\right), 31.3\left(-\mathrm{CH}_{3}\right)$; GC-MS m/z (\% relative intensity): 247 (2), $246\left(16, \mathbf{M}^{+}\right), 232$ (13), 231 (100, $\mathbf{M}^{+}-\mathbf{C H}_{3}$ ), 227 (6), 212 (7), 211 (52, $\left.\mathbf{M}^{+}-\mathbf{C H}_{3}-\mathbf{H F}\right), 183$ (13), 155 (5), 127 (7), 91 (5); IR $\left(\mathrm{KBr}, \mathrm{cm}^{-1}\right)$ 2972, 2866, 1668, 1618, 1482, 1367, 1339, 1259, 1142, 733; Anal. calc'd for $\mathrm{C}_{12} \mathrm{H}_{13} \mathrm{~F}_{3} \mathrm{O}_{2}$ : C, 58.54; H, 5.32; F, 23.15. Found: C, 58.52; H, 5.52; F, 23.36.

\section{$(R, R)-(-)-N, N^{\prime}$-Bis(5-tert-butyl-3-trifluoromethylsalicylidene)-trans-cyclohexane-}

1,2-diamine (9). (R,R)-(-)-trans-Cyclohexane-1,2-diamine (0.23 g, $2.0 \mathrm{mmol})$ was dissolved in EtOH $(15 \mathrm{ml})$ and $8(1.0 \mathrm{~g}, 4.1 \mathrm{mmol})$ was added to the solution. The resulting bright yellow solution was refluxed for $2 \mathrm{~h}$, cooled and the solvent removed in vacuo to yield a solid which was recrystallised from a water/EtOH mixture to give bright yellow crystals. $(0.84 \mathrm{~g}, 74 \%)$ : Mp 130$131^{\circ} \mathrm{C} ;\left[\alpha_{\mathrm{D}}\right]^{25}=-216\left(\mathrm{c}=1.01, \mathrm{CH}_{2} \mathrm{Cl}_{2}\right)$; IR $\left(\mathrm{KBr}, \mathrm{cm}^{-1}\right) 2967,2867,1636(\mathrm{C}=\mathrm{N}), 1606,1481$, 1367, 1333, 1285, 1261, 1127, 895, 827, 691; ${ }^{1} \mathrm{H}$ NMR $\left(300 \mathrm{MHz}, \mathrm{CDCl}_{3}\right) \delta 14.23$ (br s, 2H, $\mathrm{OH}), 8.30(\mathrm{~s}, 2 \mathrm{H}, \mathrm{N}=\mathrm{CH}), 7.54(\mathrm{~d}, 2 \mathrm{H}, J=2.1 \mathrm{~Hz}, \mathrm{ArH}), 7.31$ (d, 2H, $J=2.1 \mathrm{~Hz}, \mathrm{ArH}), 3.37-$ $3.28(\mathrm{~m}, 2 \mathrm{H}, \mathrm{C}=\mathrm{NCH}), 1.99-1.29\left(\mathrm{~m}, 8 \mathrm{H}\right.$, cyclohexyl- $\left.\mathrm{CH}_{2}\right), 1.23\left(\mathrm{~s}, 9 \mathrm{H},{ }^{\mathrm{t}} \mathrm{Bu}\right) ;{ }^{13} \mathrm{C} \mathrm{NMR}(75$ $\left.\mathrm{MHz}, \mathrm{CDCl}_{3}\right) \delta 165.2(\mathrm{C}=\mathrm{N}), 157.9,140.8,132.1(\mathrm{ArCH}), 127.0\left(\mathrm{q}, J_{\mathrm{C}-\mathrm{F}}=4.6 \mathrm{~Hz}, \mathrm{ArCH}\right), 124.1$

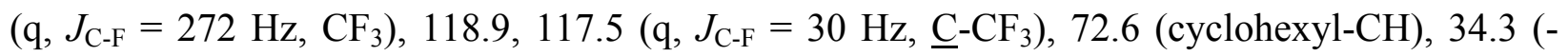
$\left.\mathrm{CMe}_{3}\right), 33.2\left(-\mathrm{CH}_{2}-\right), 31.4\left(-\mathrm{CH}_{3}\right), 24.4\left(-\mathrm{CH}_{2}-\right)$. MS-MS (Electrospray): Daughter-MS of 571 $m / z$ (\% relative intensity): $571\left(100, \mathbf{M}+\mathbf{H}^{+}\right), 551$ (30, M-F), 511 (5), 447 (5), 326 (5), 306 (5), 125 (90, $\mathbf{M}-\mathbf{C}_{23} \mathbf{H}_{23} \mathbf{F}_{6} \mathbf{O}_{2}$ ), 98 (7); Anal. calc'd for $\mathrm{C}_{30} \mathrm{H}_{36} \mathrm{~F}_{6} \mathrm{~N}_{2} \mathrm{O}_{2}$ : C, 63.15; H, 6.36; N, 4.91; F, 19.98; Found: C, 63.26; H, 6.26; N, 4.91; F, 19.90. 
CAUTION: There is evidence that $\mathrm{Cr}(\mathrm{III})$ salen and $\mathrm{O}=\mathrm{Cr}(\mathrm{V})$ salen complexes are genotoxic and carcinogenic and thus due care should be taken to avoid contact. ${ }^{56,57}$ $(R, R)-(-)-N, N^{\prime}$-Bis(5-tert-butyl-3-trifluoromethylsalicylidene)-trans-cyclohexane-1,2diamine chromium(III) chloride (10). Complex 10 was prepared using the procedure described in the supplementary information of Daly et al. ${ }^{41}$ starting from $\mathrm{CrCl}_{3}$. Ligand 9 (4.0 g, $\left.7.0 \mathrm{mmol}\right)$ gave 10 as a brown precipitate $(4.23 \mathrm{~g}, 92 \%): \mathrm{Mp}>230^{\circ} \mathrm{C}$; IR $\left(\mathrm{KBr}, \mathrm{cm}^{-1}\right) 2962,2862,1631$ $(\mathrm{C}=\mathrm{N}), 1553$, 1457, 1395, 1352, 1308, 1260, 1147, 1028, 924, 892, 864, 839, 780, 734, 697, 547; MS (Electrospray) $\mathrm{m} / z$ (\% relative intensity): 678.0 (M+Na, 5), 662.1 (15), 661.1 (M-Cl $\left.+\mathbf{H}_{\mathbf{2}} \mathbf{O}+\mathbf{N a}, 40\right), 622.2$ (10), 621.1 (40), 620.1 ( $\left.\mathbf{M - C l}^{-}, 100\right), 618.1$ (5).

[(R,R)-(-)-N,N'-Bis(5-tert-butyl-3-trifluoromethylsalicylidene)-trans-cyclohexane-1,2diamine chromium(III)] nitrate (3a). Complex 10 (500 mg, $762 \mu \mathrm{mol})$ was dissolved in $\mathrm{MeOH}$ $(20 \mathrm{ml})$ and a solution of silver nitrate $(194 \mathrm{mg}, 1.14 \mathrm{mmol})$ in water $(5 \mathrm{ml})$ was added. The resulting suspension was stirred for 30 minutes and the white precipitate of silver chloride was removed by filtration. The filtrate was concentrated under vacuum to yield a brown precipitate which was collected by filtration and dried under vacuum (445 mg, 86\%): $\mathrm{Mp}>230^{\circ} \mathrm{C}$; IR $\left(\mathrm{KBr}, \mathrm{cm}^{-1}\right)$ 2962, 2877, $1631(\mathrm{C}=\mathrm{N}), 1553,1457,1393(\mathrm{~N}-\mathrm{O}), 1259,1144,1028,924,892,864,839,781$, 734, 697, 548; MS (Electrospray) $m / z$ (\% relative intensity) 705.0 (M+Na, 3), 688.1 (5), 663.1 (10), 662.1 (15), 661.1 (M-NO $\left.\mathbf{H}_{\mathbf{2}} \mathbf{\mathbf { H } _ { 2 }} \mathbf{O}+\mathbf{N a}, 38\right), 621.1$ (57), 620.1 (M-NO $\left.\mathbf{N O}_{3}, 100\right)$.

[(R,R)-(-)- $N, N^{\prime}$-Bis(5-tertbutyl-3-trifluoromethylsalicylidene)-trans-cyclohexane-1,2diamine chromium(III)] hexafluorophosphate (3b). Complex 10 (500 mg, $762 \mu \mathrm{mol}$ ) was dissolved in the minimum amount of $\mathrm{MeOH}(20 \mathrm{ml})$ and a solution of potassium hexafluorophosphate $(211 \mathrm{mg}, 1.14 \mathrm{mmol})$ in water $(5 \mathrm{ml})$ was added. The resulting solution was stirred overnight at room temperature and concentrated to yield a brown precipitate (480 $\mathrm{mg}$, 82\%): $\mathrm{Mp}>230^{\circ} \mathrm{C}$; IR $\left(\mathrm{KBr}, \mathrm{cm}^{-1}\right)$ 2942, $1627(\mathrm{C}=\mathrm{N}), 1557,1469,1454,1397,1350,1313$, 1226, 1086, 1014, 845, 736, 559; MS (Electrospray) $\mathrm{m} / \mathrm{z}$ (\% relative intensity) $663.2(10), 662.2$ (20), 661.1 (40, $\left.\mathbf{M}_{-} \mathbf{P F}_{\mathbf{6}}+\mathbf{H}_{\mathbf{2}} \mathbf{O}+\mathbf{N a}\right), 622.2$ (10), 621.1 (50), 620.1 (M-PF 6 , 100), 618.1 (5).

General epoxidation procedure. Details of procedures for epoxidations are given in the supplementary information of Daly et al. ${ }^{41}$ Daly et al. have described conditions for the analysis of reaction mixtures by chiral GC or HPLC except: $\alpha$-methylstyrene and styrene oxide which were analysed by chiral GC (Supelco cyclodextrin- $\beta$ capillary column (betadex 120), $30 \mathrm{~m} \times$ $0.25 \mathrm{~mm}$ i.d., $0.25 \mu \mathrm{m}$ film.) with column temperatures of $80^{\circ} \mathrm{C}$ and $100^{\circ} \mathrm{C}$, respectively.

\section{Acknowledgements}

We thank Enterprise Ireland (Grants SC/97/536 and SC/01/217, scholarships for EMM, EFC), University College Dublin for Demonstratorships (EMM, EFC) and Swords Laboratories Ltd. (Bristol Myers Squibb) for a Scholarship (EMM). 


\section{References}

1. Katsuki, T. Synlett 2003, 281.

2. Katsuki, T. In Catalytic Asymmetric Synthesis; Ojima, I., Ed.; Wiley-VCH: New York, 2000; pp 287-325.

3. Jacobsen, E. N.; Wu, M. H. In Comprehensive Asymmetric Catalysis; Jacobsen, E.N.; Pfaltz, A.; Yamamoto, H. Eds; Springer: New York, 1999; Ch. 18.2, pp 649-677,

4. Abashkin, Y.G.; Burt, S. K. Org. Lett. 2004, 6, 59.

5. Cavallo, L.; Jacobsen, H. J.Org. Chem. 2003, 68, 6202.

6. Cavallo, L.; Jacobsen, H. Eur. J. Inorg. Chem. 2003, 892.

7. Ivanic, J. J. Chem. Phys. 2003, 119, 9377.

8. Khavrutskii, I.V.; Musaev, D.G.; Morokuma, K. Inorg. Chem. 2003, 42, 2606.

9. Feth, M. P.; Bolm, C.; Hildebrand, J. P.; Kohler, M.; Beckmann, O.; Bauer, M.; Ramamonjisoa, R.; Bertagnolli, H. Chem. Eur. J. 2003, 9, 1348.

10. Bryliakov, K. P.; Talsi, E. P. Inorg.Chem. 2003, 42, 7258.

11. Kureshy, R. I.; Khan, N. H.; Abdi, S. H. R.; Ahmad, I.; Singh, S.; Jasra, R.V. J. Catal. 2004, 221, 234.

12. Kureshy, R. I.; Khan, N. H.; Abdi, S. H. R.; Ahmed, I.; Singh, S.; Jasra, R.V. J. Mol. Catal. A 2003, 203, 69.

13. Kureshy, R. I.; Khan, N. H.; Abdi, S. H. R.; Singh, S.; Ahmed, I.; Shukla, R. S.; Jasra, R.V. J. Catal. 2003, 219, 1.

14. Sellner, H.; Karjalainen, J.K.; Seebach, D. Chem. Eur. J. 2001, 7, 2873.

15. Leadbeater, N. E.; Marco, M. Chem. Rev. 2002, 102, 3217.

16. McNamara, C.A.; Dixon, M.J.; Bradley, M. Chem. Rev. 2002, 102, 3275.

17. Prasad, J. S.; Vu, T.; Totleben, M. J.; Crispino, G. A.; Kacsur, D.J.; Swaminathan, S.; Thornton, J. E.; Fritz, A.; Singh, A. K. Org. Process Res. Dev. 2003, 7, 821.

18. Lane, B. S.; Burgess, K. Chem. Rev. 2003, 103, 2457.

19. Bräse, S.; Lauterwasser, F.; Ziegert, R. E. Adv. Synth. Catal. 2003, 345, 869.

20. Jacobsen, E. N. Acc. Chem. Res. 2000, 33, 421.

21. Haufe, G.; Bruns, S. Adv. Synth. Catal. 2002, 344, 165.

22. Bandini, M.; Cozzi, P. G.; Melchiore, P.; Umani-Ronchi, A. Angew.Chem., Int. Ed. 2004, 43, 84.

23. Schaus, S. E.; Branalt, J.; Jacobsen, E. N. J. Org. Chem. 1998, 63, 403.

24. Aikawa, K.; Irie, R.; Katsuki, T. Tetrahedron 2001, 57, 845.

25. Mihara, J.; Aikawa, K.; Uchida, T.; Irie, R.; Katsuki, T. Heterocycles 2001, 54, 395.

26. Bandini, M.; Cozzi, P. G.; Umani-Ronchi, A. Chem. Commun. 2002, 919.

27. Gianneschi, N. C.; Bertin, P. A.; Nguyen, S. T.; Mirkin, C. A.; Zakharov, L. N.; Rheingold, A.L. J. Am. Chem. Soc. 2003, 125, 10508.

28. Paddock, R. L.; Nguyen, S. T. J. Am. Chem. Soc. 2001, 123, 11498. 
29. Darensbourg, D. J.; Yarbrough, J.C.; Ortiz, C.; Fang, C.C. J. Am. Chem. Soc. 2003, 125, 7586.

30. Bukowska, A.; Bukowski, W.; Noworol, J. J. Mol. Catal. A 2003, 203, 95.

31. Dioos, B. M. L.; Jacobs, P.A. Tetrahedron Lett. 2003, 44, 4715.

32. Dioos, B. M. L.; Jacobs, P.A. Tetrahedron Lett. 2003, 44, 8815.

33. Haufe, G.; Bruns, S.; Runge, M. J. Fluorine Chem 2001, 112, 55.

34. Samsel, E.G.; Srinivasan, K.; Kochi, J. K. J. Am. Chem. Soc. 1985, 107, 7606.

35. Imanishi, H.; Katsuki, T. Tetrahedron Lett. 1997, 38, 251.

36. Bousquet, C.; Gilheany, D.G. Tetrahedron Lett. 1995, 36, 7739.

37. Dalton, C.T.; Ryan, K.M.; Wall, V.M.; Bousquet, C.; Gilheany, D.G. Top. Catal. 1998, 5, 71.

38. For a notable exception see: Nishikori, H.; Ohta, C.; Katsuki, T. Synlett 2000, 1557.

39. For the conversion of cis-alkenes to trans-epoxides see: Chang, S.; Galvin, J. M.; Jacobsen, E.N. J. Am. Chem. Soc. 1994, 116, 6937.

40. Ryan, K. M.; Bousquet, C.; Gilheany, D. G. Tetrahedron Lett. 1999, 40, 3613.

41. Daly, A. M.; Renehan, M.F.; Gilheany, D.G. Org. Lett. 2001, 3, 663.

42. O'Mahony, C. P.; McGarrigle, E. M.; Renehan, M. F.; Ryan, K. M.; Kerrigan, N. J.; Bousquet, C.; Gilheany, D.G. Org. Lett. 2001, 3, 3435.

43. McGarrigle, E. M.; Murphy, D.M.; Gilheany, D.G. Tetrahedron: Asymmetry 2004, 15, 1343.

44. Stokker, G. E.; Deana, A. A.; deSolms, S. J.; Smith, R. L.; Cragoe, E. J. J.; Baer, J. E.; Ludden, C. T.; Russo, H. F.; Scriabine, A.; Sweet, C. S.; Watson, L. S. J. Med. Chem. 1980, $23,1414$.

45. Christensen, H. Synth .Commun. 1975, 5, 65.

46. Strekowski, L.; Lin, S.-Y.; Lee, H.; Wydra, R. L.; Kiselyov, A. S. Heterocycl. Commun. 1997, 3, 109.

47. Kobayashi, Y.; Kumadaki, I. Acc. Chem. Res. 1978, 11, 197.

48. Meprathu, B. V.; Protasiewicz, J. D. ARKIVOC 2003, (vi), 83.

49. Linde, C.; Koliaï, N.; Norrby, P.O.; Akermark, B. Chem. Eur. J. 2002, 8, 2568.

50. Adam, W.; Roschmann, K. J.; Saha-Moller, C. R.; Seebach, D. J. Am. Chem. Soc. 2002, 124, 5068.

51. Wang, S. H.; Mandimutsira, B. S.; Todd, R.; Ramdhanie, B.; Fox, J. P.; Goldberg, D. P. J. Am. Chem. Soc. 2004, 126, 18.

52. Collman, J. P.; Zeng, L.; Decreau, R. A. Chem. Commun. 2003, 2974.

53. Nam, W.; Choi, S. K.; Lim, M.H.; Rohde, J.-U.; Kim, I.; Kim, J.; Kim, C.; Que, J R. L. Angew. Chem., Int. Ed. 2003, 42, 109.

54. Schanz, H.-J.; Linseis, M.; Gilheany, D.G. Tetrahedron: Asymmetry 2003, 14, 2763.

55. Saltzmann, H.; Sharefkin, J.G. Org. Synth. Coll. 1973, 5, 658.

56. Dillon, C. T.; Lay, P.A.; Bonin, A.M.; Dixon, N. E.; Sulfab, Y. Aust. J. Chem. 2000, 53, 411.

57. Bose, R. N.; Fonkeng, B. F. Chem. Commun. 1996, 2211. 